\title{
THE
}

$5-19-1980$

\section{Observation of Quasibound States of the Neutron in Matter}

\author{
K.-A. Steinhauser
}

A. Steyerl

University of Rhode Island, asteyerl@uri.edu

H. Scheckenhofer

S. S. Malik

University of Rhode Island, smalik@uri.edu

Follow this and additional works at: https://digitalcommons.uri.edu/phys_facpubs

Terms of Use

All rights reserved under copyright.

\section{Citation/Publisher Attribution}

Steinhauser, K.-A., Steyerl, A., Scheckenhofer, H., \& Malik, S. S. (1980). Observation of Quasibound States of the Neutron in Matter. Physical Review Letters, 44(20), 1306-1309. doi: 10.1103/PhysRevLett.44.1306 Available at: http://dx.doi.org/10.1103/PhysRevLett.44.1306

This Article is brought to you for free and open access by the Physics at DigitalCommons@URI. It has been accepted for inclusion in Physics Faculty Publications by an authorized administrator of DigitalCommons@URI. For more information, please contact digitalcommons-group@uri.edu. 


\title{
Observation of Quasibound States of the Neutron in Matter
}

\author{
K.-A. Steinhauser, A. Steyerl, and H. Scheckenhofer \\ Fakultät für Physik, Technische Universität München, D-8046 Garching, Germany \\ and \\ S. S. Malik \\ Fakultät für Physik, Technische Universität München, D-8046 Garching, Germany, and Physics Department, \\ University of Rhode Island, Kingston, Rhode Island 02881 \\ (Received 29 February 1980)

\begin{abstract}
Quasistationary states of ultracold neutrons have been observed in a double-hump potential barrier. This potential barrier was created by sandwiching a thin film of aluminum between two thin copper films. Measurements of reflection from and transmission through such composite films displayed clear resonances. The resonance positions and widths are in agreement with calculations.
\end{abstract} \\ PACS numbers: $14.20 . \mathrm{Cg}$
}

This paper reports unambiguous observations of the existence of quasistationary states of the neutron in matter. That the effective nuclear scattering potential could lead to such states was first pointed out by Kagan. ${ }^{1}$ Seregin ${ }^{2}$ subsequently proposed that an arrangement of thin films (with appropriate thickness and made of materials having different values of the effective nuclear potential) could constitute a double-hump potential barrier in which neutrons with energies $\sim 10^{-7} \mathrm{eV}$ could form quasibound states. The quantum characteristics of states in such a potential region can be obtained by solving a one-dimensional Schrödinger equation involving only the neutron wave-vector component, $k_{z}$, perpendicular to the potential barrier. In semiclassical (WKB) approximation, the values of $k_{z}$ for which such states are formed are given by

$$
k_{z} \cong(\pi / d)\left(n+\frac{1}{2}\right),
$$

where $d$ is the width of the potential well enclosed by the two humps, and $n$ is a positive integer including 0. Also,

$$
k_{z}=\left[2 m\left(E_{n}-V\right)\right]^{1 / 2} / \hbar,
$$

where $m$ denotes the neutron mass, $E_{n}$ the kinetic energy corresponding to the neutron velocity component in $z$ direction, and

$$
V=2 \pi \hbar \mathrm{Nb} / \mathrm{m} \text {, }
$$

the scattering potential at the bottom of the well. $N$ and $b$, respectively, are the number density (in inverse cubic centimeters) and the coherentscattering length of the medium.

In the experiment reported here, values of $k_{z}$ could be varied with use of the "gravity diffractometer" 3,4 for ultracold neutrons. As shown in
Fig. 1, the ultracold neutron beam produced by the neutron turbine facility ${ }^{5}$ at the Forschungsreaktor Munich (Garching) is guided (via neutron guides) to the entrance slit system of the gravity diffractometer. The diffractometer, in brief, consists of entrance and exit slit systems (slits of $2 \mathrm{~cm}$ width together with beam stops) and three neutron mirrors, two vertical and one horizontal. The entrance and exit slit height corresponds to the maxima of the neutron flight parabolae for the incoming and exiting beam. In these conditions the entrance slit is imaged on the exit slit. The vertical position of the horizontal mirror (measured from the slit height, or, more precisely, from the center of gravity of the maxima for the set of incident neutron trajectories capable of passing the system) measures the fall height, $z$, of the neutron. The value of $k_{z}$, then, is given by

$$
k_{z}=(m / \hbar)(2 g z)^{1 / 2},
$$

where $g$ is the acceleration due to gravity.

In the normal operation of the gravity diffractometer, shown in Fig. 1, the center of the first vertical mirror, $A$, was fixed at fall heights between 50 and $60 \mathrm{~cm}$. The height, $z$, of the horizontal mirror, $B$, was varied. In order to maintain the focusing condition, the horizontal position of the second vertical mirror, $C$, requires specific adjustment. The reflected intensity was measured using a $\mathrm{BF}_{3}$ detector depleted in ${ }^{10} \mathrm{~B}$ content and arranged $80 \mathrm{~cm}$ below the exit slit. Thus, the neutrons gain enough energy in the gravitational field to be able to penetrate the thin detector window $(0.1 \mathrm{~mm} \mathrm{Al})$.

A measurement of beam intensity with horizontal mirror, $B$, as nickel-coated glass mirror of $10 \mathrm{~cm} \times 20 \mathrm{~cm}$ yielded 45 counts per hour (about 


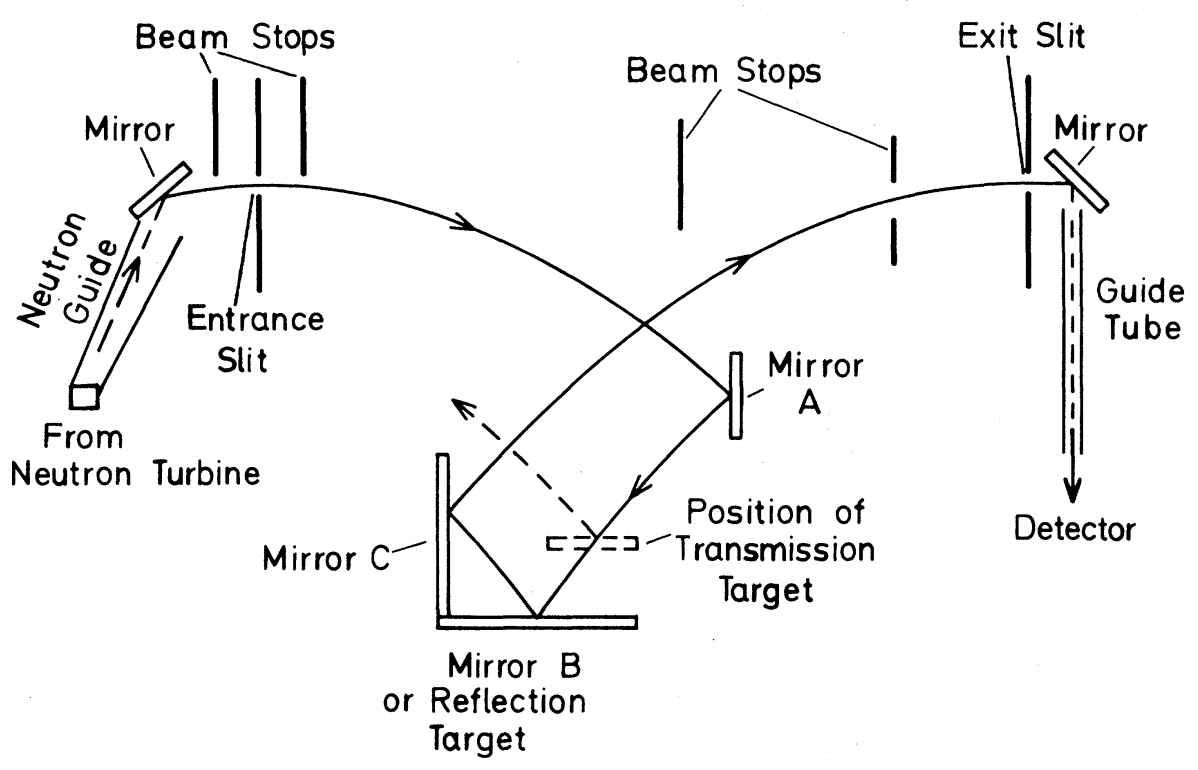

FIG. 1. Experimental arrangement showing the positions of the multilayered targets. For reflection measurements, the target acts as the horizontal mirror, $B$, of the mirror system of the gravity diffractometer. The entrance and exit slit (together with beam stops) define and analyze the ultracold neutron beam. A change in the fall height is achieved by a change in vertical target position.

one-half of the value measured previously ${ }^{3,4}$ ) with a small negative slope $(0.18$ counts $/ \mathrm{h} \mathrm{cm})$ at higher values of $z$. The dispersion in the value of $k_{z}$, convoluted with the analyzer (exit) slit resolution as determined by the slit width, was measured by varying the vertical exit slit position. The full width at half maximum (FWHM) of the resolution curve determined in this way was 3 $\mathrm{cm}$, the same as in Refs. 3 and 4 and consistent with the value $\Delta k_{z} / k_{z}=\frac{1}{2} \Delta z / z \cong 1 \%$ obtained by a Monte Carlo simulation of neutron passage through the instrument. ${ }^{4}$

The properties of the neutron beam reflected from a double-hump potential barrier were studied by replacing the mirror $B$ by a target of the same size and consisting of thin films of aluminum and copper deposited on glass substrate. The double-hump potential well is created by sandwiching an Al film (nominal thickness $860 \AA$ ) between two $\mathrm{Cu}$ films of $240 \AA$ each. These films were evaporated on the glass substrate one at a time, monitoring the thickness by the frequency of a quartz crystal. During the evaporation the substrate was continuously rotated in such a way as to limit variations of film thickness over the sample extension to $<3 \%$. The outer $\mathrm{Cu}$ layer was protected from oxidation by an additional $100-\AA-$ thick $\mathrm{Al}$ film. The total thickness of the layers determined by an optical-interference microscope was found to agree with the quartz-crystal data.

The intensity reflected from the target measured as a function of fall height is shown in Fig. 2. The data show a clear reflection minimum at $z=108.5 \mathrm{~cm}$. The solid line in Fig. 2 represents the calculation of the reflected intensity obtained by solving the Schrödinger equation for the multistep potential region due to the $\mathrm{Al}-\mathrm{Cu}-\mathrm{Al}-\mathrm{Cu}-$ glass media, as depicted in the inset of Fig. 2. A thickness of $881 \pm 2 \AA$ was required for the width of the potential well between the two humps for $n=1$ (second level) for the position of the calculated minimum to coincide with the observed value at $108.5 \mathrm{~cm}$, in reasonable agreement with the optical microscope and quartz-oscillator data of $860 \AA$. The fitted value $225 \pm 5 \AA$ for the $\mathrm{Cu}$ film thickness is slightly less than the nominal value of $240 \AA$. The observed width (FWHM) of $7.5 \mathrm{~cm}(7.7 \mathrm{neV})$ corresponds to a lifetime of the state of the order of $\hbar / \Gamma=2 \times 10^{-7} \mathrm{~s}$. The depth of the minimum, should reach almost the background level of $12.4 \pm 0.4$ counts $/ \mathrm{h}$. There is only a slight enhancement due to the instrumental resolution of $2 \mathrm{neV}$. The observations, however, indicate a noticeable gap between the calculated minimum $13.8 \pm 0.4$ counts $/ \mathrm{h}$ and the observed value $17.4 \pm 0.7$ counts $/ \mathrm{h}$. Possible reasons for this discrepancy are discussed later.

A second target with $\mathrm{Cu}-\mathrm{Al}-\mathrm{Cu}$ film thicknesses 


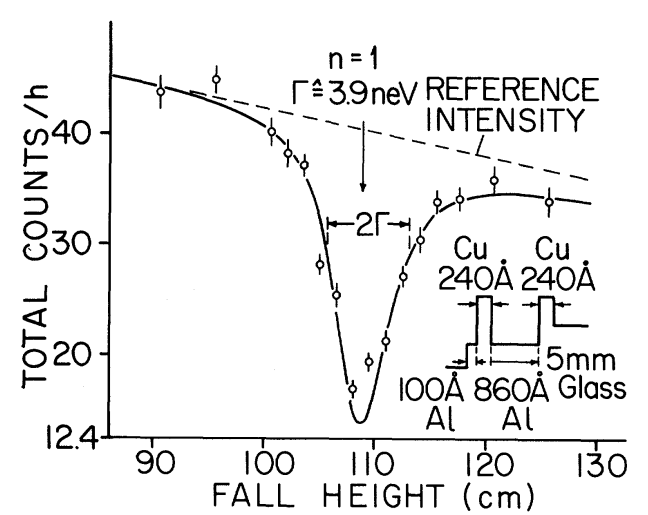

FIG. 2. Reflected intensity measured as a function of the fall height for a target with nominal layer thicknesses: $\mathrm{Al}(100 \AA), \mathrm{Cu}(240 \AA), \mathrm{Al}(860 \AA)$, and $\mathrm{Cu}(240 \AA)$. The substrate is glass. Potential functions representing these layers are shown in the inset. The intensity minimum at $108.5 \mathrm{~cm}$ corresponds to $n=1$ (the second stationary state). The solid curve is an exact solution of the one-dimensional Schrödinger equation for the multistep potential representing the target and includes the instrumental resolution broadening.

selected to allow observation of the third stationary state $(n=2)$ showed similar reflection characteristics.

A transmission target was prepared by evaporation of $\mathrm{Al}(110 \AA), \mathrm{Cu}(180 \AA), \mathrm{Al}(1670 \AA), \mathrm{Cu}$ $(180 \AA)$, and $\mathrm{Al}(110 \AA)$ on a substrate assembled from eight well-polished $5 \times 5-\mathrm{cm}^{2}$ silicon crystals of $0.25-\mathrm{mm}$ thickness. Silicon was chosen because of its low absorption cross section and because it can be polished well down to sufficiently small thicknesses to achieve a transparency of $\sim 70 \%$ in the wavelength range investigated. This target was installed $16 \mathrm{~cm}$ above mirror $B$ (Fig. 1), thus enabling separation of the transmitted and reflected beams. The measured transmission data are shown in Fig. 3. The data show two resonances $(n=1$ and $n=2)$. The line positions $(74$ and $99 \mathrm{~cm})$ and widths $(3.4$ and $5.9 \mathrm{~cm})$ are fairly well represented by the solid fitted curve for the following layer thicknesses: central $\mathrm{Al}$ layer, $1663 \pm 3 \AA$; Cu barriers, $190 \pm 5 \AA$. The step in the transmission curve at $z=85 \mathrm{~cm}$ is due to different optimal settings of mirror $A$ (Fig. 1), and consequently different incident beam intensities, at lower and higher fall heights. As in the reflection measurements, a discrepancy with expectation is observed in the resonance intensity, since the transmission maxima are lower than calculated.

The Breit-Wigner width for the $n$th quasista-

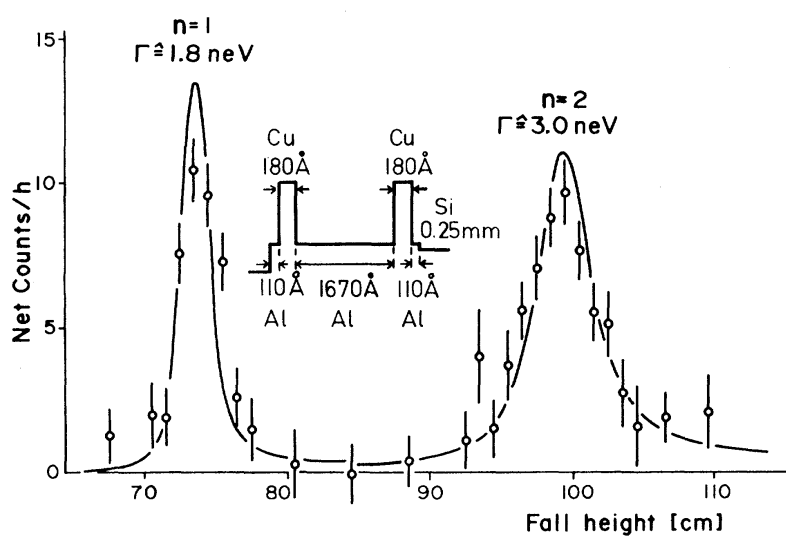

FIG. 3. Transmission data for a target with nominal layer thicknesses: Al (110 ̊), Cu (180 ̊), Al (1670 ̊), $\mathrm{Cu}(180 \AA)$, and Al $(110 \AA)$. The substrate is silicon of $0.25-\mathrm{mm}$ thickness. The two resonances observed correspond to $n=1$ and $n=2$. The data are compared to the solid curve calculated for a multistep potential.

tionary state at $E=E_{n}$ depends upon the probability, $P$, of tunneling through a potential barrier. In quasiclassical (WKB) approximation,

$$
P=\exp \left\{-2 d_{b}\left[2 m\left(V_{b}-E_{n}\right)\right]^{1 / 2} / \hbar\right\},
$$

where $V_{b}$ and $d_{b}$ are the height and width of the barrier, respectively. For identical barriers defining the double hump the resonance lines are of nearly Lorentzian shape with a FWHM of $2 \Gamma^{*}$ $=\hbar \omega P / \pi$, where $\hbar \omega \simeq E_{n+1}-E_{n}{ }^{2,6}$ For instance, for the resonance of Fig. 2 the respective calculated WKB width is $2 \Gamma^{*}=2.2 \mathrm{neV}$. The exact treatment yields the value $2 \Gamma=5.1 \mathrm{neV}$ (for the nominal $\mathrm{Cu}$ film thickness) which agrees better with the measured value $7.7 \mathrm{neV}$. The difference can be explained if $\mathrm{Cu}$ film thickness is reduced $(225 \AA$ instead of $240 \AA)$. Similar deviations between exact calculation and measurement were observed for the other resonances investigated.

As noted earlier, the experimental resonance extrema for all the targets are lower than the corresponding calculated and resolution broadened values. A poorer resolution than the $2 \mathrm{neV}$ employed in the calculations can explain the difference. However, exit-slit scans at the position of reflectivity minimum and at the wings yielded a resolution (FWHM) of $\sim 3 \mathrm{~cm}$ in agreement with the instrumental resolution. Unequal transparencies of the two potential barriers $\left(P_{A} \neq P_{B}\right)$ would lead to nonzero reflectivity minima (and transmission maxima $<1$ ), but the data would require $\sim 20 \%$ deviations between $P_{A}$ and $P_{B}$ which are unlikely to exist. Departures from the rectangular 
potential shape (due to diffusion mixing at the Al$\mathrm{Cu}$ boundaries) would result in changes of level position and width, but not in reduced resonance amplitudes. Thus, a slight blurring of the potential boundaries by $\sim 80 \AA$ would eliminate the small mismatch (by $2 \%$ ) of theoretical and experimental level spacings in Fig. 3. A variation in layer thickness of $\sim 3 \%$ is, however, insufficient to account for the reduced resonance amplitudes. Microscopic density fluctuations of surface microroughness would give rise to disorder scattering and, consequently, coherent-beam attenuation, similar to "absorption" (including nuclear capture and thermal inelastic scattering). Calculation shows that an enhancement of "absorption" in pure $\mathrm{Al}$ by a factor of $\sim 10^{2}-10^{3}$ could explain the data, but such strong scattering is unlikely to exist.

The optical analog of the double-hump potential barrier is the well-known Fabry-Perot interferometer. Thus, the present results indicate that a neutron Fabry-Perot interferometer with a resolution of $10^{-9} \mathrm{eV}$ is feasible.
The authors are deeply indebted to Mr. Fuhrmann of Agfa Corporation (Munich) for his expert and timely help in preparation of the targets. Capable and sustained assistance from $\mathrm{Mr}$. Nagel and Mr. Schreiber is gratefully acknowledged. One of us (S.S.M.) deeply appreciates the hospitality of Professor W. Gläser during his stay at Munich and the travel assistance from the University of Rhode Island. This research was supported in part by the German Bundesministerium für Forschung und Technologie.

\footnotetext{
${ }^{1}$ Yu. Kagan, Pis'ma Zh. Eksp. Teor. Fiz. 11, 235 (1970) [JETP Lett. 11, 147 (1970)].

${ }^{2}$ A. A. Seregin, Zh. Eksp. Teor. Fiz. 73, 1634 (1977) [Sov. Phys. JETP $\underline{46}, 859$ (1977)].

${ }^{3}$ H. Scheckenhofer and A. Steyerl, Phys. Rev. Lett. $\underline{39}$, 1310 (1977).

${ }^{4} \mathrm{H}$. Scheckenhofer, Dissertation, Technische Universität München, 1979 (unpublished).

${ }^{5}$ A. Steyerl, Nucl. Instrum. Methods 125, 461 (1975).

${ }^{6}$ A. Steyerl, T. Ebisawa, K.-A. Steinhauser, and M. Utsuro, "Neutron optics with simple multilayer systems" (to be published).
}

\title{
High-Energy Photoproduction of the $D^{*+}$
}

\author{
P. Avery, J. Wiss, J. Butler, (a) G. Gladding, M. C. Goodman, (b) \\ T. O'Halloran, J. J. Russell, and A. Wattenberg \\ University of Illinois, Urbana, Illinois 61801
}

and

M. Binkley, J. Cumalat, I. Gaines, M. Gormley, R. L. Loveless, ${ }^{(c)}$ and J. Peoples

Fermi National Accelerator Laboratory, Batavia, Ilinois 60510

and

M. S. Atiya, and S. D. Holmes, B. C. Knapp, W. Lee, and W. J. Wisniewski

Columbia University, New York, New York 10027

(Received 28 February 1980)

Photoproduction of the $D^{*+}$ have been observed where the $D^{*+}$ decays via $D^{*+} \rightarrow \pi^{+} D^{0}$ and the $D^{0}$ decays via $K^{*} \pi^{+}$and $K_{s} \pi^{+} \pi^{-}$. The $D^{*+}, D^{0}$ mass difference and the $D^{0}$ mass observed here are in excellent agreement with previous measurements of charmed mesons produced in $e^{+} e^{-}$annihilations. The photoproduced $D^{*+}$ and $D^{*-}$ signals are produced nearly equally at the level of about $100 \mathrm{nb} /$ nucleon.

PACS numbers: 14.40.Pe, 13.60.Kd

We report on the observation of the $D^{*+}\left(D^{*-}\right)$ produced in the wideband neutral beam at Fermilab. Both this beam and our detector have been described in a previous Letter. ${ }^{1}$ Our exposure consisted of approximately $6 \times 10^{11}$ photons with an energy greater than $50 \mathrm{GeV}$ incident on a 2cm-long scintillator target. The particles emerg- ing from these interactions were analyzed by a large-acceptance magnetic spectrometer system (multiwire proportional chambers) which includes two multicell Cherenkov counters with pion threholds of 6 and $12 \mathrm{GeV}$, a large lead-glass array for neutral detection, and a hadron calorimeter. The data described here were recorded under a 